(2) Open Access Full Text Article

ORIGINALRESEARCH

\title{
Downregulation of miR-200a Protects Mouse Leydig Cells Against Triptolide by Triggering Autophagy
}

Hui Miao

Congxiu Miao

Jing Han

$\mathrm{Na} \mathrm{Li}$

Department of Reproduction and Genetics, Heping Hospital Affiliated to Changzhi Medical College, Changzhi, Shanxi 046000, People's Republic of China
This article was published in the following Dove Press journal: Drug Design, Development and Therapy

Background: MicroRNAs play important roles in testicular development and spermatogenesis. Previous research has indicated that the level of miR-200a was significantly upregulated in patients with different spermatogenic impairments. However, the mechanism by which miR-200a regulated spermatogenic impairments remains unclear.

Methods: Leydig cells were treated with triptolide (TP) to mimic spermatogenic impairments. CCK-8 and flow cytometry were used to detect the proliferation and apoptosis in Leydig cells, respectively. In addition, Western blot assay was used to examine ATG7, ATG5, p62 protein levels in MLTC-1 cells.

Results: TP dose-dependently upregulated the expression of miR-200a in MLTC-1 cells. In addition, TP inhibited the proliferation of MLTC-1 cells via inducing apoptosis and oxidative stress; however, these phenomena were notably reversed by miR-200a antagomir. Furthermore, luciferase reporter assay identified that ATG7 was the direct binding target of miR-200a. TP treatment markedly inhibited the activation of autophagy in MLTC-1 cells via inhibition of ATG7. Conversely, downregulation of miR-200a significantly induced autophagy in TP-treated MLTC-1 cells by activation of ATG7. Meanwhile, the cell protective effects of miR-200a against TP were reversed by autophagy inhibitor 3MA, indicating that autophagy plays an important role.

Conclusion: These results indicated that downregulation of miR-200a could protect MLTC1 cells against TP by inducing autophagy. Therefore, miR-200a might serve as a new therapeutic target for the treatment of male hypogonadism.

Keywords: hypogonadism, triptolide, microRNA-200a, autophagy, apoptosis

\section{Introduction}

Hypogonadism is a condition characterized by testosterone deficiency and impairment of spermatogenesis. ${ }^{1}$ Hypogonadism affects approximately $6 \%$ of males. ${ }^{2}$ Male reproduction ability mainly relies on androgens, which are largely secreted by testicular Leydig cells. ${ }^{3}$ Testosterone is a steroid hormone, which is mainly produced by Leydig cells. ${ }^{4}$ Moreover, hypogonadism often leads to some comorbidities, such as obesity, insulin resistance (IR), type-2 diabetes mellitus (T2DM), and skeletal muscle wasting. ${ }^{5}$ Meanwhile, the number of Leydig cells in the adult human testis will reduce with increasing age. ${ }^{6}$ Therefore, improving the treatment of hypogonadism contributes to maintaining the sexual function of males.

Triptolide (TP) was isolated from Tripterygium wilfordii Hook F, which is a diterpenoid triepoxide. ${ }^{7}$ There is evidence that TP exhibits anti-tumor,
Correspondence: Congxiu Miao Genetics, Heping Hospital Affiliated to Changzhi Medical College, I 10 South Yan'an Road, Changzhi, Shanxi 046000, People's Republic of China Email miaocongxiu321@I26.com 
immunosuppressive and anti-inflammatory properties, ${ }^{7,8}$ and also that impairment of spermatogenesis is associated with longer treatment duration with TP. ${ }^{9}$ In addition, Lue et al. found that TP could induce infertility in the adult rats, indicating that TP could increase the deformity rate of sperm. ${ }^{10}$

MicroRNAs (miRNAs) are a class of endogenous noncoding RNA molecules with 18-22 nucleotides. ${ }^{11}$ MiRNAs can directly bind to the 3'UTR region of its target genes, and regulate the expression of targeted genes. ${ }^{12}$ In addition, miRNAs participate in various pathological processes including cell differentiation, cell survival, apoptosis, and protein metabolism. ${ }^{13}$ Evidence has shown that some miRNAs are abundantly expressed in various type of male germ cells. ${ }^{14}$ Abu-Halima et al. found that the level of miR-200a was notably upregulated in patients with different spermatogenic impairments. ${ }^{15}$ However, the mechanism by which miR-200a regulated spermatogenic impairments remains unclear. Hence, this study aimed to investigate the role of miR-200a in spermatogenic impairments using an in vitro cell model.

\section{Materials and Methods}

\section{Cell Culture and Cell Transfection}

The mouse leydig MLTC-1 and TM3 cell lines were purchased from American Type Culture Collection (ATCC, Rockville, MD, USA). Cells were cultured in complete media (DMEM medium containing 10\% fetal bovine serum and 1\% penicillin/streptomycin) and incubated in an incubator with $5 \% \mathrm{CO}_{2}$ at $37^{\circ} \mathrm{C}$. The passage number of cell lines was 3 .

MiR-200a agomir and miR-200a antagomir were obtained from GenePharma (Shanghai, China). MLTC-1 or TM3 cells were plated into 6 -well plates at a density of $4 \times 10^{5}$ cells per well overnight at $37^{\circ} \mathrm{C}$. Then, cells were transfected with miR-200a agomir or miR-200a antagomir for $24 \mathrm{~h}$ at $37^{\circ} \mathrm{C}$ using Lipofectamine 2000 reagent (Thermo Fisher Scientific, Waltham, MA, USA) according to the manufacturer's instructions.

\section{Real-Time Quantitative Polymerase Chain Reaction (RT-qPCR)}

Total RNA was extracted using RNAiso Plus (Takara Biotechnology, Co. Ltd, Dalian, China) according to the provided protocol. Then, the PrimeScript ${ }^{\mathrm{TM}}$ RT Master Mix Kit (Takara Biotechnology) was used to synthesize complementary DNA. After that, RT-qPCR was performed using a SYBR Green ${ }^{\mathrm{TM}}$ Premix Ex Taq $^{\mathrm{TM}}$ (Takara
Biotechnology). The primers used were as follows: U6: forward, 5'-CTCGCTTCGGCAGCACAT-3'; reverse, 5'AACGCTTCACGAATTTGCGT-3'. MiR-200a: forward, 5'-GGAGTTCGTATCGGCTGCGATG-3'; reverse, 5'CGACCGTGTAATCGTCGTTGC-3'. The level of miR200a was normalized to that of U6.

\section{Cell Counting Kit-8 (CCK-8)}

TP (purity: 99.79\%) was purchased from MedChem Express (Monmouth Junction, NJ, USA). TP was dissolved in DMSO $(10 \mathrm{mM})$ first; the store solution of TP was diluted to the indicated concentration with DMEM. MLTC- 1 or TM3 cells ( $5 \times 10^{4}$ cells per well) were plated onto 96 -well plates and incubated overnight at $37^{\circ} \mathrm{C}$. After that, cells were treated with TP $(30,90,120$ or $150 \mathrm{nM})$ for $24 \mathrm{~h}$. Later on, cells were incubated with $10 \mu \mathrm{L} \mathrm{CCK} 8$ reagent (Beyotime, Shanghai, China) at $37^{\circ} \mathrm{C}$ for another 2 $h$. The absorbance was measured at a wavelength of 450 nm using a microplate reader (Bio-Rad Laboratories, Inc., Hercules, CA, USA). The dose of TP was calculated based on previous studies. ${ }^{16,17}$

\section{Flow Cytometric Analysis}

Apoptosis in MLTC-1 and in TM3 cells was detected by an Annexin V-FITC/PI Apoptosis Detection Kit (SigmaAldrich, St. Louis, MO, USA). Cells were washed three times with pre-cold PBS, and then were resuspended in $500 \mu \mathrm{L}$ of binding buffer. After that, cells were stained with $5 \mu \mathrm{L}$ of propidium iodide (PI) and $5 \mu \mathrm{L}$ of Annexin V-FITC at room temperature for $15 \mathrm{~min}$ in the dark. Subsequently, the number of annexin V-FITC-positive cells was determined with a FACS flow cytometer (BD Biosciences, Franklin Lakes, NJ, USA).

\section{Trypan Blue Exclusion Assay}

For evaluation of cell death, MLTC-1 cells were incubated with $0.25 \%$ trypan blue solution (Gibco, Paisley, UK). Subsequently, viable cells were counted using a hemacytometer (Neubauer improved, Marienfeld, Germany).

\section{Dual-Luciferase Reporter Assay}

The fragment of ATG7 3'-UTR containing the binding site of miR-200a was inserted into the pmirGLO dualLuciferase vector, named wild-type (WT)-ATG7. The vector pmirGLO-200a-MT (mutant) was inserted by the ATG7-MT, which contains point mutations of the miR200a seed region binding site. After that, MLTC-1 cells were co-transfected with WT-ATG7 or MT-ATG7 plasmid, 
with miR-200a agomir respectively using Lipofectamine 2000. After $48 \mathrm{~h}$ of incubation, the luciferase activity in cell lysate was detected using the Dual Luciferase Reporter Assay System (Promega, Madison, WI, USA) according to the manufacturer's protocol.

\section{Immunofluorescence Assay}

MLTC-1 cells were fixed with PBS containing 4\% paraformaldehyde for $1 \mathrm{~h}$, and then permeabilized with $0.1 \%$ Triton X-100 for $30 \mathrm{~min}$. After that, cells were blocked with $1 \%$ bovine serum albumin for $2 \mathrm{~h}$, and then incubated overnight with an antibody against LC3B $(1: 100 ; 10 \mu \mathrm{g} / \mathrm{mL}$, Abcam Cambridge, MA, USA) at $4^{\circ} \mathrm{C}$. Later on, cells were incubated with horseradish peroxidase-conjugated secondary antibody (1:1000, Abcam) at room temperature for $1 \mathrm{~h}$, and then observed under a confocal microscopy (Olympus CX23 Tokyo, Japan). The nucleus was stained with DAPI for $5 \mathrm{~min}$. Autophagy inhibitor 3-Methyladenine (3MA) was purchased from Sigma Aldrich (St. Louis, MO, USA).

\section{ROS Analysis}

Intracellular ROS levels of MLTC-1 cells were determined by staining of cells with $2^{\prime}, 7^{\prime}$-dichlorodihydrofluorescein diacetate (DCFH-DA; Sigma Aldrich). The fluorescence was detected by flow cytometry as described in previous studies. $^{18,19}$

\section{Western Blot Assay}

Total proteins were quantified using a BCA protein assay kit (Beyotime Institute of Biotechnology, Beijing, China). Protein lysates (30 $\mu \mathrm{g}$ loading) were separated by $10 \%$ sodium dodecyl sulfate-polyacrylamide gel electrophoresis. After that, the gels were transferred onto polyvinylidene difluoride (PVDF) membrane (Millipore, Billerica, MA, USA), followed by blocking with $5 \%$ skimmed milk in TBST for $1 \mathrm{~h}$ at room temperature. Later on, the membrane was incubated in primary antibodies against ATG7 (1:1000), p-ERK (1:1000), ERK (1:1000), ATG5 (1:1000), p62 (1:1000), Bax (1:1000), Bcl-2 (1:1000), active caspase 3 (1:1000), active caspase 9 (1:1000), LC3B (1:1000) and $\beta$ actin $(1: 1000)$ at $4^{\circ} \mathrm{C}$ overnight, followed by incubating with a secondary antibody (1:5000). Subsequently, signals were visualized using a KeyGEN Enhanced ECL detection kit (KeyGEN, Nanjing, China). All antibodies were obtained from Abcam (Cambridge, MA, USA). The concentration of primary antibodies used in Western blot was $1 \mu \mathrm{g} / \mathrm{mL}$.
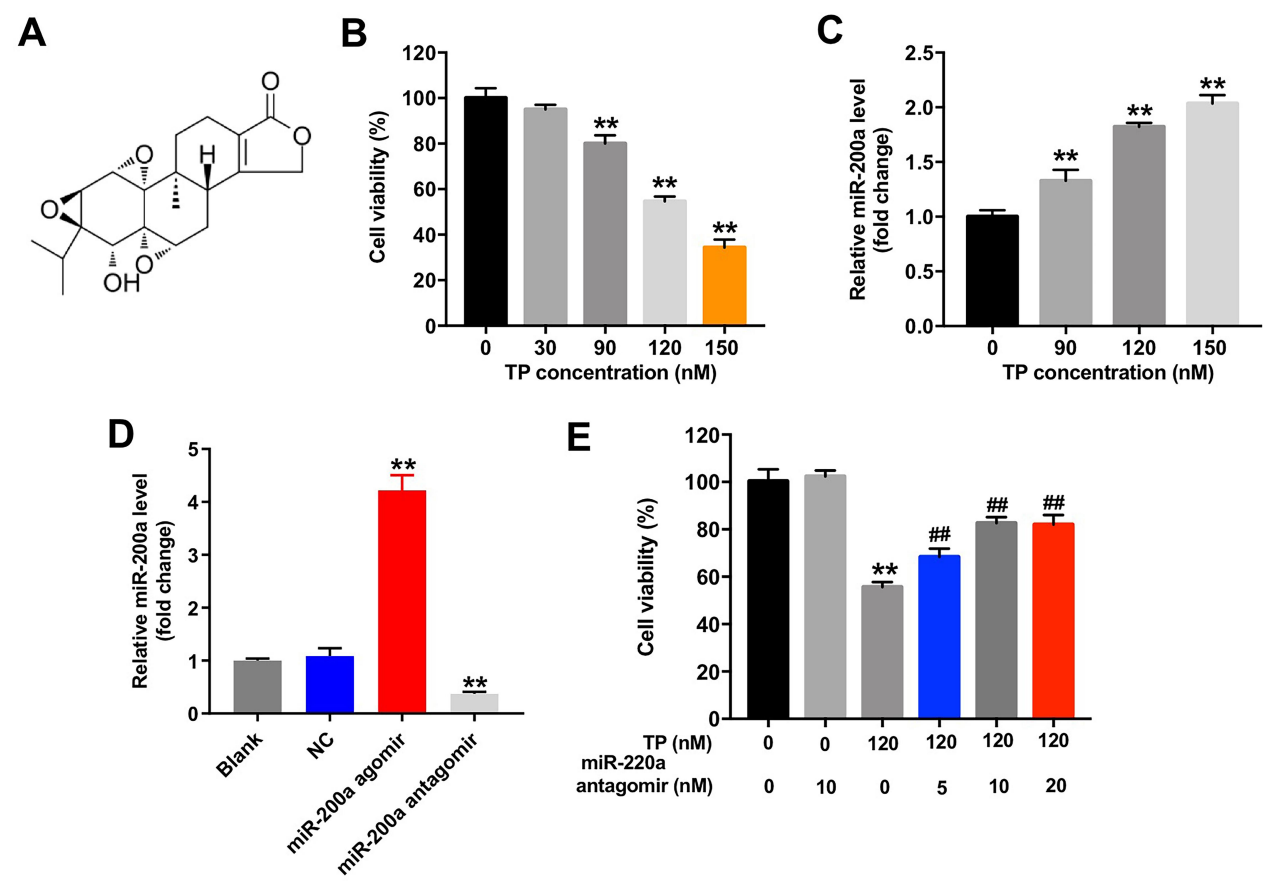

Figure I Downregulation of miR-200a attenuated TP-induced cytotoxicity in MLTC-I cells. (A) The chemical structure of triptolide. (B) MLTC-I cells were treated with TP $(30,90,120$, or $150 \mathrm{nM})$ for $24 \mathrm{~h}$. CCK-8 assay was used to detect cell viability. (C) MLTC-I cells were treated with TP (90, I20, or I50 nM) for 24 h. RT-qPCR was used to determine the level of miR-200a in cells. (D) MiR-200a level in MLTC-I cells transfected with miR-200a agomir or miR-200a antagomir was analyzed by qRT-PCR. (E) MLTCI cells were transfected with miR-200a antagomir (5, I0, or $20 \mathrm{nM}$ ) first, and then exposed to $120 \mathrm{nM}$ TP for $24 \mathrm{~h}$. CCK-8 assay was used to detect the cell viability. These experiments were repeated three times. $* * \mathrm{P}<0.01$ compared with control group; ${ }^{\#} \mathrm{P}<0.01$ compared with TP (I $\left.20 \mathrm{nM}\right)$ group. Abbreviations: TP, triptolide; CCK-8, Cell Counting Kit-8; NC, negative control. 


\section{ELISA Assay}

ELISA kits (Nanjing Jiancheng Bioengineering Institute, Jiangsu, China) were used to detect the levels of MDA, SOD, GSH in cell culture supernatants.

\section{Statistical Analysis}

All data were repeated in triplicate. Data are presented as the mean \pm standard deviation (S.D.). All statistical analyses were performed using GraphPad Prism software (version 7.0, La Jolla, CA, USA). The results of CCK-8, RT-qPCR, trypan blue exclusion, immunofluorescence, Western blot, flow cytometry, ROS detection and ELISA assays were analyzed by one-way analysis of variance (ANOVA) followed by Tukey's tests (multiple comparisons). In addition, the result of dualluciferase reporter assay was analyzed by two-way ANOVA followed by Tukey's tests (multiple comparisons). ${ }^{*} \mathrm{P}<0.05$ was considered to be statistically significant.

\section{Results}

\section{Downregulation of miR-200a Attenuated} TP-Induced Cytotoxicity in Leydig Cells

To assess the effect of TP on the proliferation of MLTC-1 cells, CCK-8 assay was used. The chemical structure of TP is shown in Figure 1A and CCK-8 data indicated TP inhibited the proliferation of MLTC-1 cells in a dose dependent manner (Figure 1B). In addition, the level of miR-200a was significantly upregulated in TP-treated cells (Figure 1C). As expected, miR-200a agomir markedly increased the level of miR-200a in MLTC-1 cells, while miR-200a antagomir exhibited a completely opposing effect (Figure 1D). Furthermore, downregulation of miR200a attenuated the cytotoxicity of TP on MLTC- 1 and TM-3 cells (Figure 1E and Supplementary Figure 1A). These data indicated that downregulation of miR-200a could attenuate TP-induced cytotoxicity in Leydig cells.

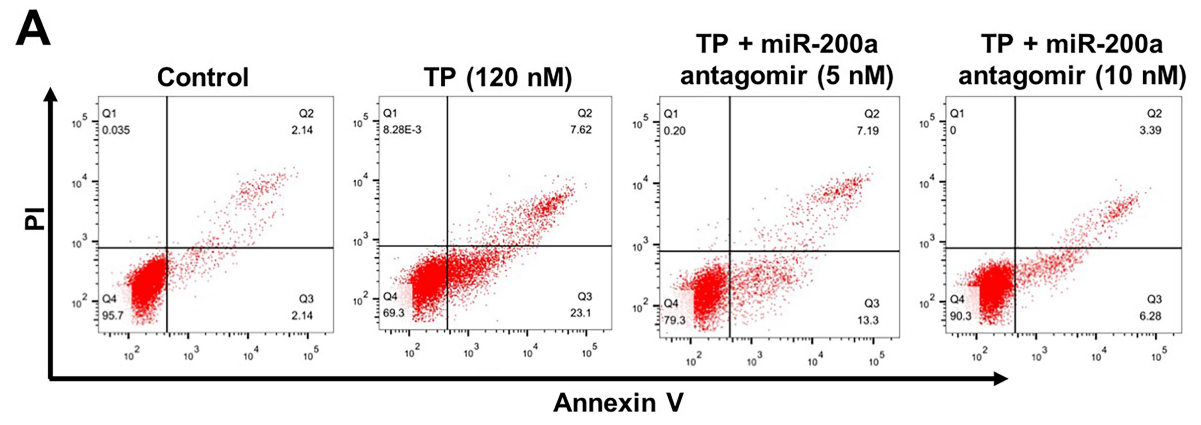

B
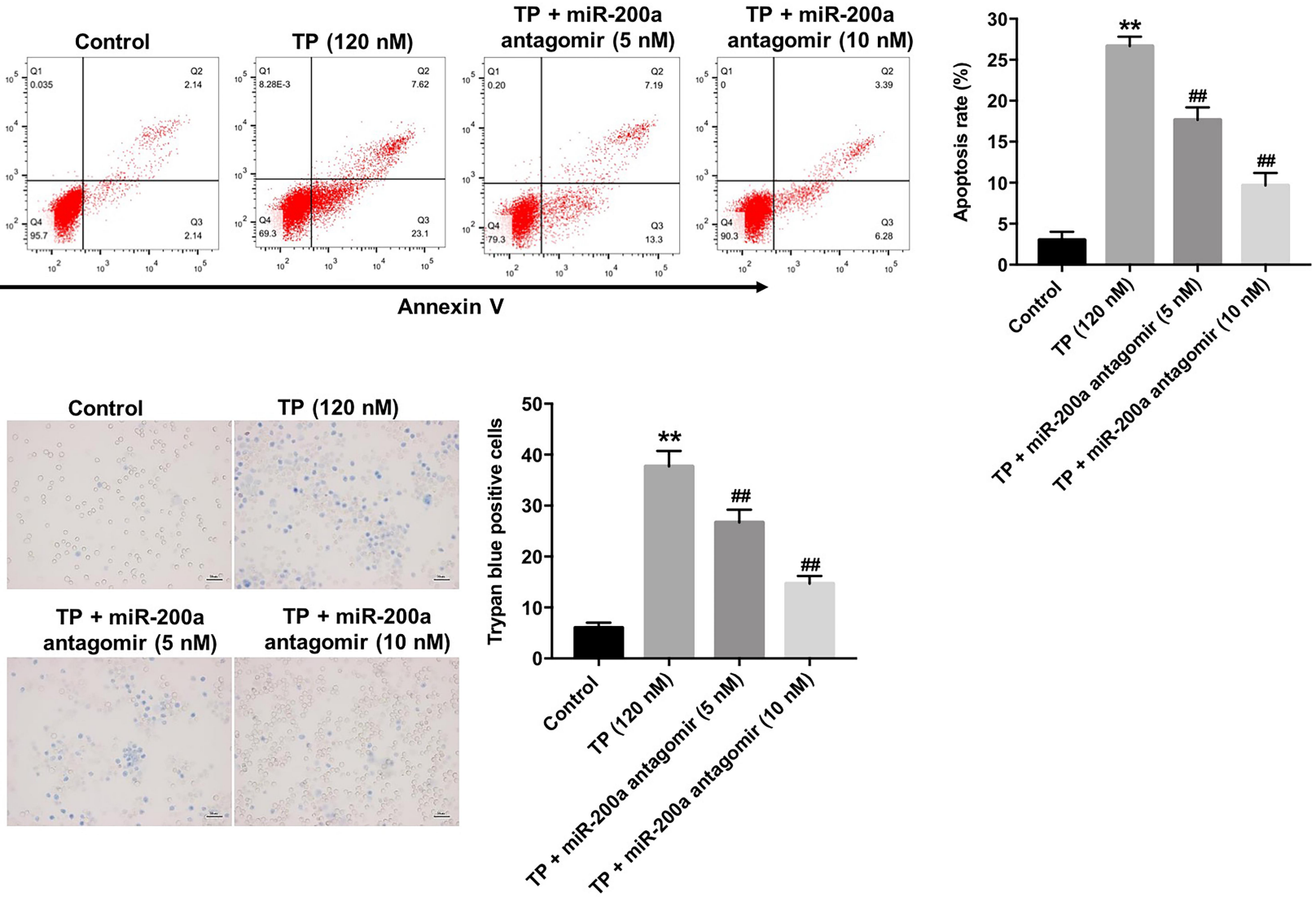

Figure 2 Downregulation of miR-200a attenuated TP-induced cell death in MLTC-I cells. MLTC-I cells were transfected with miR-200a antagomir (0, 5, or I0 nM) first, and then exposed to $120 \mathrm{nM}$ TP for $24 \mathrm{~h}$. (A) Apoptotic cells were measured with Annexin V and PI double staining. (B) Trypan blue staining assay was used to detect cell death. These experiments were repeated three times. ${ }^{*} * \mathrm{P}<0.0$ I compared with control group; ${ }^{\# \#} \mathrm{P}<0.0$ I compared with TP (I20 nM) group.

Abbreviations: TP, triptolide; PI, propidium iodide. 


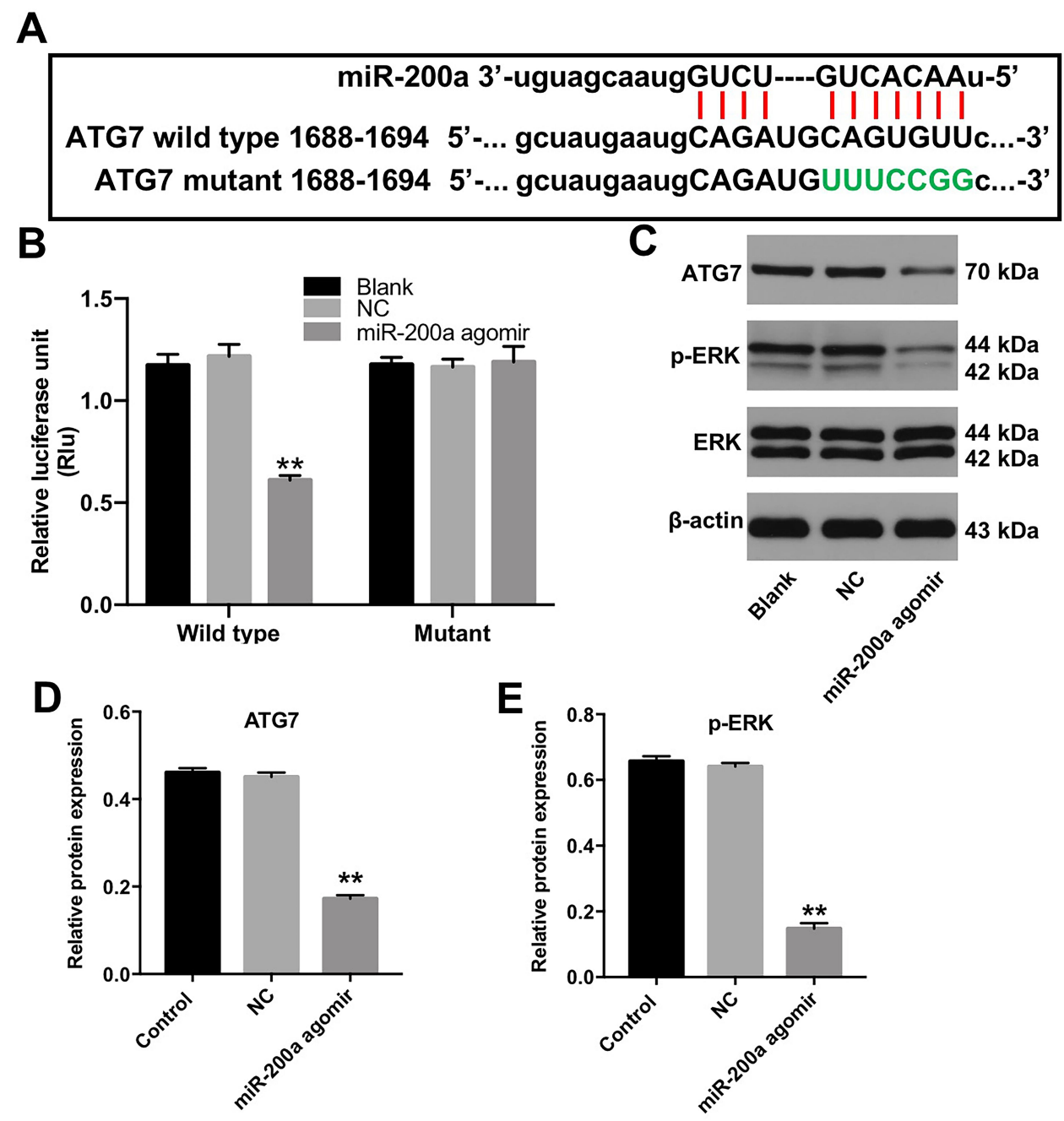

Figure 3 ATG7 was a direct binding target of miR-200a. (A) The putative binding sites of miR-200a on ATG7 and the mutated sequences are shown. (B) Luciferase activity in MLTC-I cells following co-transfection with ATG7-WT/MT 3'-UTR plasmid and miR-200a agomir was measured using dual luciferase reporter assay. (C) Western analysis of ATG7 and p-ERK protein levels in MLTC-I cells. (D and E) The relative expressions of ATG7 and p-ERK in MLTC-I cells were quantified by normalizing to $\beta$-actin and ERK. These experiments were repeated three times. ${ }^{*} * \mathrm{P}<0.01$ compared with the NC group.

Abbreviation: NC, negative control.

\section{Downregulation of miR-200a Attenuated TP-Induced Apoptosis of Leydig Cells}

To further explore the protective effect of miR-200a antagomir in TP-treated Leydig cells, flow cytometry was applied. As indicated in Figure 2A and supplemen tary Figure 1B, TP significantly induced apoptosis of Leydig cells, which was notably reversed after transfection with $10 \mathrm{nM}$ miR-200a antagomir. In addition, trypan blue staining assay indicated that downregulation of miR-200a markedly blocked TP-induced cell death in Leydig cells (Figure 2B and supplementary Figure $1 \mathrm{C}$ ). These data suggested that downregulation of miR-200a could attenuate TP-induced cell death in Leydig cells.

\section{ATG7 Was a Direct Binding Target of miR-200a}

The online bioinformatics tools miRDB (http://www.mirdb. org) and microRNA (http://www.microrna.org/microrna/ home.do) were used to identify the target genes of miR200a. As shown in Figure 3A, miR-200a had a complementary sequence to ATG7. In addition, dualluciferase reporter assay indicated that miR-200a agomir suppressed the luciferase activity of ATG7-WT in MLTC-1 cells, but it did not affect the luciferase activity of ATG7-MT (Figure 3B). In addition, miR-200a agomir notably decreased the expressions of ATG7 and phosphorylation of ERK in MLTC-1 cells (Figure 3C-E). All these results illustrated that ATG7 was a direct binding target of miR-200a. 


\section{Downregulation of miR-200a Triggered} Autophagy in TP-Treated MLTC-I Cells

To investigate MLTC-1 cell autophagy, immunofluorescent assay was used. As shown in Figure 4A, TP significantly inhibited the formation of LC3B puncta in MLTC-1 cells, indicating that TP could inhibit autophagy. In contrast, downregulation of miR-200a increased the accumulation of LC3B in TP-treated MLTC-1 cells, compared with TP treatment group; however, that effect was abolished in the presence of 3MA (Figure 4A). In addition, Western blot data indicated that TP decreased the expressions of ATG7, ATG5 and LC3B II and increased the level of p62 in MLTCcells, while these phenomena were reversed by miR-200a antagomir (Figure 4B-F). However, the inductive effect of miR-200a antagomir on autophagy in TP-treated MLTC-1 cells was reversed by $3 \mathrm{MA}$ (Figure 4B-F). These data suggested that downregulation of miR-200a could trigger autophagy in TP-treated MLTC-1 cells.

\section{Downregulation of miR-200a Attenuated TP-Induced Oxidative Stress in MLTC-I \\ Cells via Inducing Autophagy}

We next explored the effect of miR-200a antagomir on oxidative stress in TP-treated MLTC-1 cells by detecting the relevant biomarkers. As shown in Figure 5A-D, TP markedly increased the levels of ROS and MDA, and decreased the activities of SOD and GSH in MLTC-1 cells; however, these TP-induced changes were reversed by miR-200a antagomir.

We next investigated whether cell autophagy was involved in these processes. As indicated in Figure 5A-D, the inhibitory effects of miR-200a antagomir on oxidative stress in TP-treated MLTC-1 cells were significantly

A
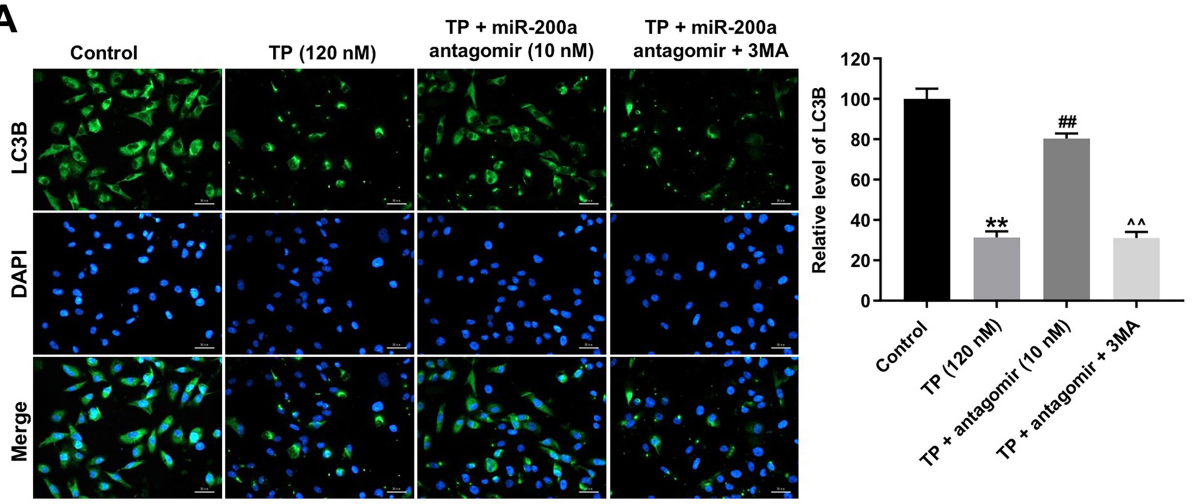

C

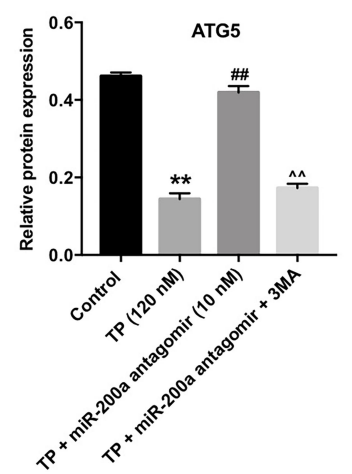

D

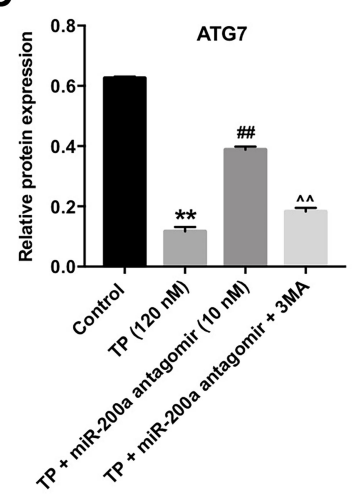

E

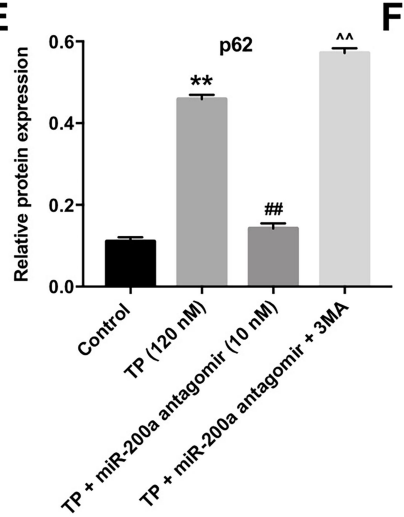

B

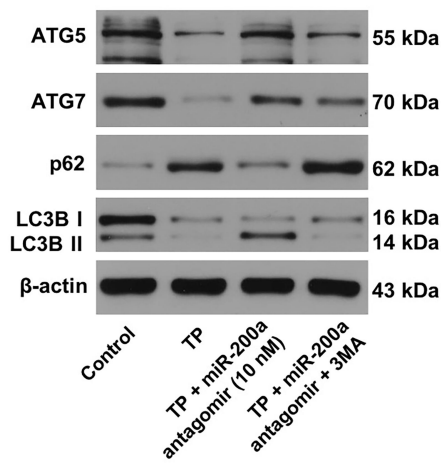

$F$

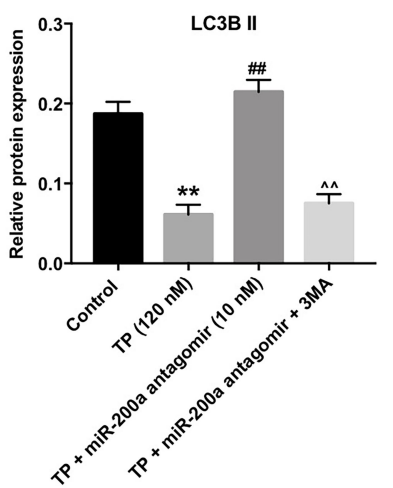

Figure 4 Downregulation of miR-200a triggered autophagy in TP-treated MLTC-I cells. MLTC-I cells were transfected with $10 \mathrm{nM}$ miR-200a antagomir first, and then exposed to $120 \mathrm{nM}$ TP for $24 \mathrm{~h}$. Cells were treated with $5 \mathrm{mM} 3 \mathrm{MA}$ prior to $12 \mathrm{~h}$ of the miR-200a antagomir treatment. (A) LC3B puncta were observed by immunofluorescence using a confocal microscope. (B) Western analysis of ATG7, ATG5, p62 and LC3B protein levels in MLTC-I cells. (C-F) The relative expressions of ATG7, ATG5, p62 and LC3B in MLTC-I cells were quantified by normalizing to $\beta$-actin. These experiments were repeated three times. $* * P<0.01$ compared with control group; ${ }^{\#} \mathrm{P}<0.01$ compared with TP $(120 \mathrm{nM})$ group; ${ }^{\wedge} \mathrm{P}<0.01$ compared with TP + miR-200a antagomir group.

Abbreviations: TP, triptolide; 3MA, 3-methyladenine. 


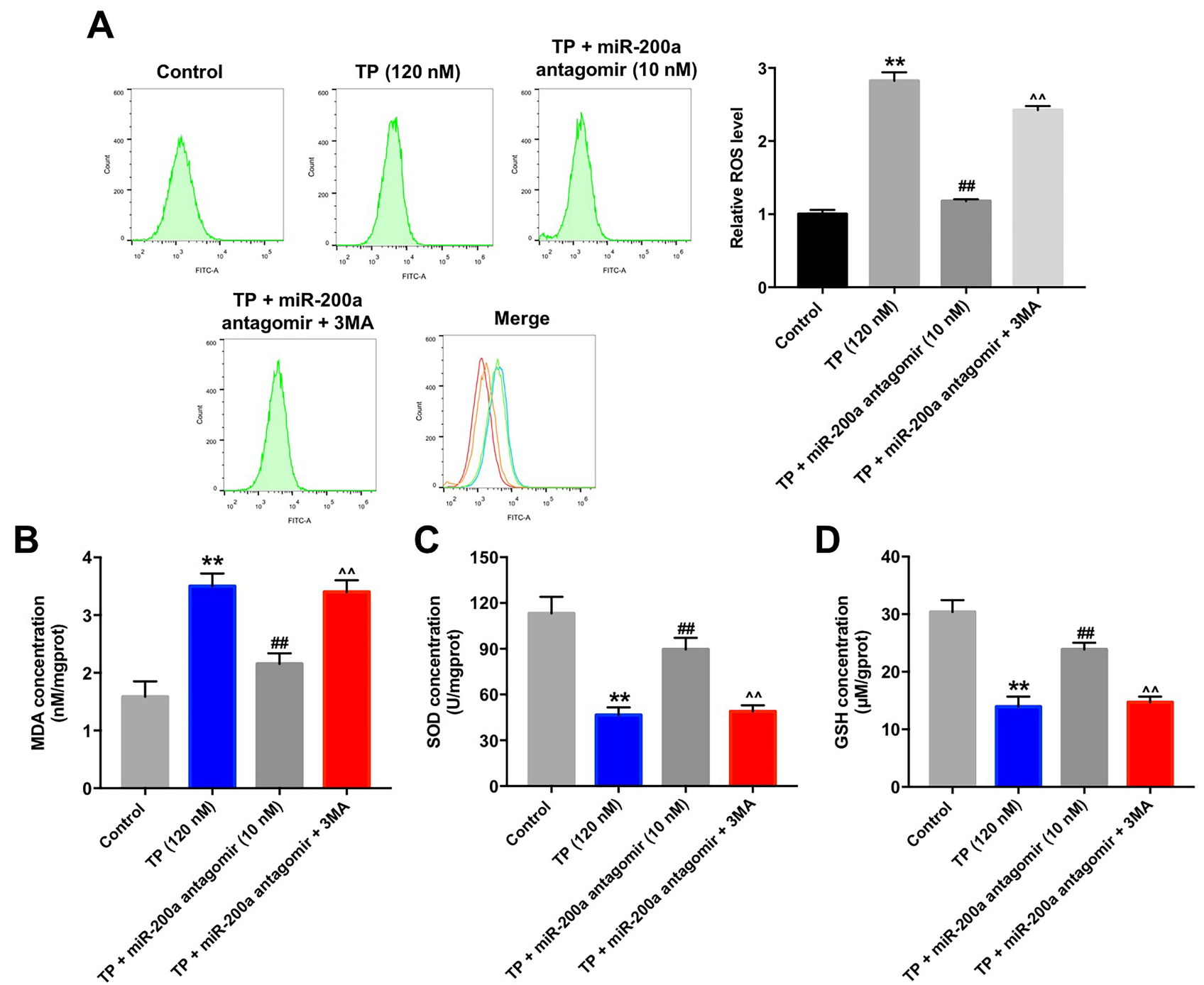

Figure 5 Downregulation of miR-200a attenuated TP-induced oxidative stress in MLTC-I cells via inducing autophagy. MLTC-I cells were transfected with I0 nM miR-200a antagomir first, and then exposed to $120 \mathrm{nM}$ TP for $24 \mathrm{~h}$. Cells were treated with $5 \mathrm{mM}$ 3MA prior to $12 \mathrm{~h}$ of the miR-200a antagomir treatment. (A) Intracellular ROS generation was assessed by flow cytometry. (B-D) Levels of MDA, SOD, and GSH in cells were detected with ELISA. These experiments were repeated three times. ${ }_{* * \mathrm{P}<0.01}$ compared with control group; ${ }^{\#} \mathrm{P}<0.01$ compared with TP $(120 \mathrm{nM})$ group; ${ }^{\wedge} \mathrm{P}<0.01$ compared with TP + miR-200a antagomir group.

Abbreviations: TP, triptolide; 3MA, 3-methyladenine.

abolished by $3 \mathrm{MA}$. These data indicated that downregulation of miR-200a could attenuate TP-induced oxidative stress in MLTC-1 cells via inducing autophagy.

\section{Downregulation of miR-200a Attenuated TP-Induced Apoptosis in MLTC-I Cells via Inducing Autophagy}

Since miR-200a antagomir could inhibit apoptosis and induce autophagy in TP-treated MLTC-1 cells, we sought to investigate the interaction between autophagy and apoptosis. As shown in Figure 6A, treatment with 3MA significantly abolished the protective effect of miR-200a antagomir in TP-treated MLTC-1 cells. In addition, the inhibitory effect of miR-200a antagomir on apoptosis in TP-treated MLTC-1 cells was abolished by the treatment with 3MA (Figure 6B). Western blot data showed the effect of miR-200a antagomir on the expressions of Bax, active caspase 3, active caspase 9, and Bcl-2 was reversed by $3 \mathrm{MA}$ as well (Figure $6 \mathrm{C}-\mathrm{G}$ ). All these results revealed that downregulation of miR-200a could attenuate TP-induced apoptosis in MLTC-1 cells via inducing autophagy.

\section{Discussion}

Evidence has shown that TP could result in degenerative changes in seminiferous tubules and epididymides and lead to a significant reduction in the weights of testis and epididymis. $^{20}$ In addition, TP could induce reproductive 


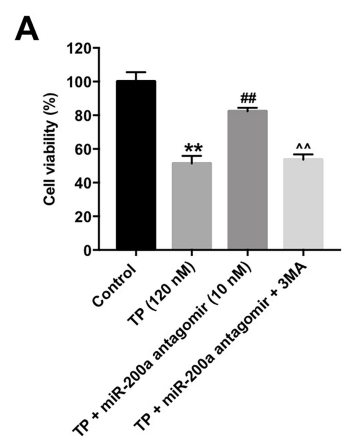

C

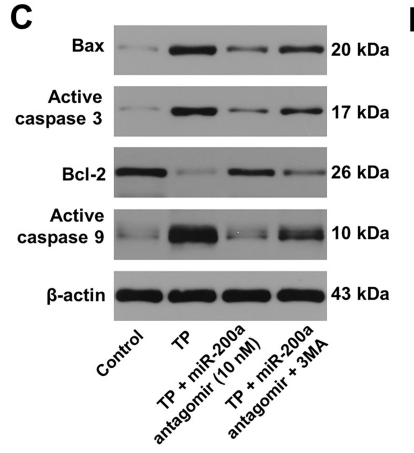

B

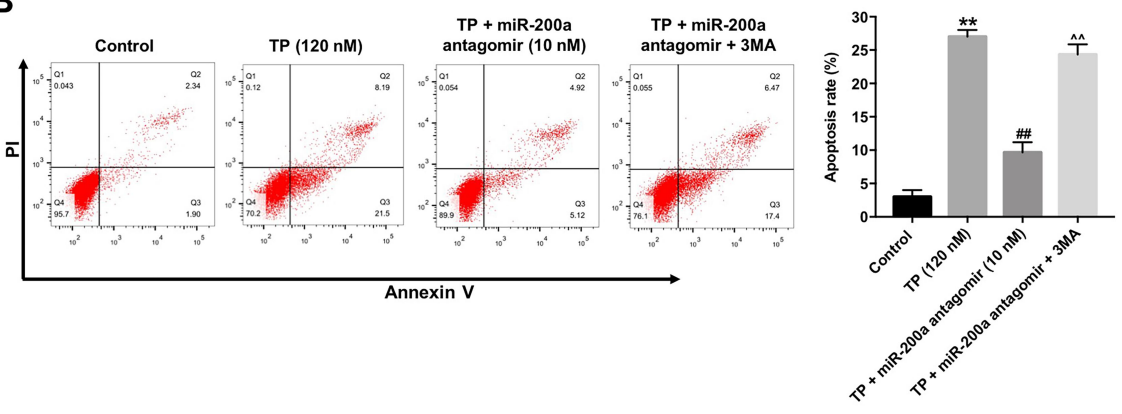

D E

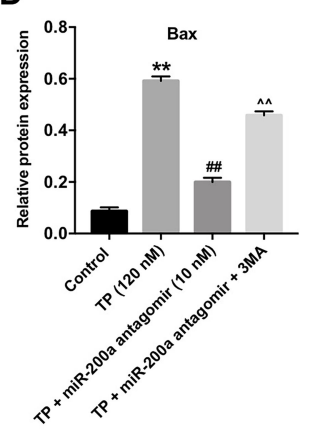

E

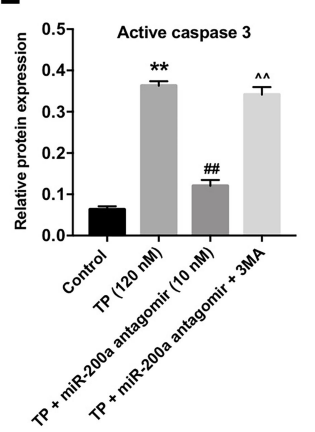

$\mathbf{F}$

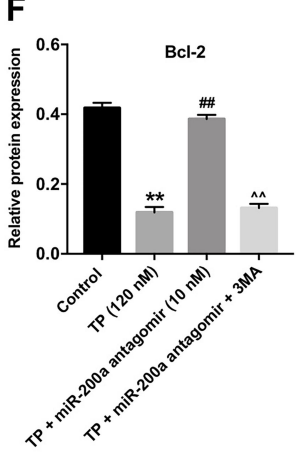

G

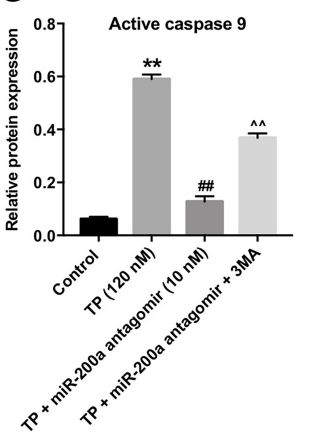

Figure 6 Downregulation of miR-200a attenuated TP-induced apoptosis in MLTC-I cells via inducing autophagy. MLTC-I cells were transfected with 10 nM miR-200a antagomir first, and then exposed to $120 \mathrm{nM}$ TP for $24 \mathrm{~h}$. Cells were treated with $5 \mathrm{mM} 3$ MA prior to $12 \mathrm{~h}$ of the miR-200a antagomir treatment. (A) CCK-8 assay was used to detect cell viability. (B) Apoptotic cells were measured with Annexin $\mathrm{V}$ and PI double staining. (C) Western analysis of Bax, active caspase 3 , Bcl-2 and active caspase 9 protein levels in MLTC-I cells. (D-G) The relative expressions of Bax, active caspase 3, Bcl-2 and active caspase 9 in MLTC-I cells were quantified by normalizing to $\beta$-actin. These experiments were repeated three times. ${ }^{* * P}<0.0$ I compared with control group; ${ }^{\# P} \mathrm{P}<0.01$ compared with TP $(I 20 \mathrm{nM})$ group; ${ }^{\wedge} \mathrm{P}<0.0 \mathrm{I}$ compared with TP + miR200a antagomir group.

Abbreviations: TP, triptolide; 3MA, 3-methyladenine; CCK-8, Cell Counting Kit-8; PI, propidium iodide.

toxicity in Leydig cells via triggering apoptosis and accumulation of ROS. ${ }^{21}$ Excessive amounts of ROS can result in lasting oxidative stress and can induce cell apoptosis. ${ }^{22,23} \mathrm{In}$ addition, oxidative stress could affect normal spermatozoa production by impairing the steroidogenic capacity of the testis. ${ }^{22}$ As we know, MDA is used as an important biomarker for examining oxidative stress, while SOD and GSH are important antioxidants. ${ }^{24}$ In this study, we found that TP significantly increased the levels of ROS and MDA, and decreased the activities of SOD and GSH in MLTC-1 cells. Meanwhile, TP markedly increased the expressions of Bax and active caspase 3 in MLTC- 1 cells. These data indicated that TP significantly induced apoptosis and oxidative stress in MLTC-1 cells, which were consistent with previous studies. ${ }^{21,25}$ Xiong et al. found that ectopic expression of miR-200a significantly inhibited motility traits of sperm. ${ }^{26}$ Our data found that downregulation of miR-200a inhibited apoptosis and oxidative stress in TP-treated MLTC-1 cells, indicating that miR-200a may play a critical role in the development of hypogonadism.

MiRNAs have been found to exert their functions by suppressing the expression of their target genes. ${ }^{27}$ Our data identify ATG7 as a binding target of miR-200a. ATG7 is an important autophagy-related protein, which could increase autophagy. ${ }^{28}$ Autophagy, as a self-digestion process, can maintain intracellular homeostasis and contribute to cell survival. ${ }^{29}$ In this study, miR-200a agomir significantly downregulated the expressions of ATG7 and phosphorERK. Gao et al. found that dysfunction of autophagy could reduce testosterone production in some patients with oligospermia. ${ }^{30}$ In addition, inhibition of autophagy could induce oxidative stress and reduce testosterone levels. ${ }^{31}$ In the current study, TP significantly inhibited autophagy in MTLC-1 cells via downregulation of ATG7, ATG5 and LC3B, which was consistent with previous research. ${ }^{32}$ Conversely, downregulation of miR-200a increased the expressions of LC3B, ATG7 and ATG5, but decreased the expression of p62 in TP-treated Leydig cells, thereby promoting cell autophagy. However, the effects of miR-200a antagomir were abolished by 3MA. All these data indicated that miR-200a antagomir could active the autophagy in TPtreated MTLC-1 cells by targeting ATG7. Nevertheless, it is possible that miR-200a regulated other targets in this setting and more investigations are needed in future. 
The cytoprotective function of autophagy can inhibit cell apoptosis and reduce ROS accumulation. ${ }^{33}$ Our data indicated that downregulation of miR-200a could protect Leydig cells from TP-induced apoptosis and oxidative stress. However, the inhibitory effects of miR-200a antagomir on apoptosis and oxidative stress in TP-treated MLTC-1 cells were reversed by the treatment with 3MA. These data indicated that downregulation of miR-200a could ameliorate apoptosis and oxidative stress in TPtreated MLTC-1 cells via triggering autophagy.

Male hypogonadism is the result of deficiency of the hormone testosterone. ${ }^{34}$ The Leydig cells are responsible for producing testosterone, and testosterone is critical for spermatogenesis. ${ }^{35}$ Thus, in the future, it is important to investigate whether miR-200a could affect the level of hormone testosterone in TP-treated MLTC-1 cells.

\section{Conclusion}

Our study demonstrates that downregulation of miR-200a protects against $\mathrm{TP}$-induced reproductive toxicity via targeting ATG7, suggesting that miR-200a might serve as new therapeutic target for the treatment of male hypogonadism.

\section{Funding}

This work was supported by Shanxi Health Commission (No. 2018125).

\section{Disclosure}

The authors declare no competing financial interests.

\section{References}

1. Pivonello R, Menafra D, Riccio E, et al. Metabolic disorders and male hypogonadotropic hypogonadism. Front Endocrinol (Lausanne). 2019;10:345. doi:10.3389/fendo.2019.00345

2. Leisegang $\mathrm{K}$, Henkel $\mathrm{R}$. The in vitro modulation of steroidogenesis by inflammatory cytokines and insulin in TM3 Leydig cells. Reprod Biol Endocrinol. 2018;16(1):26. doi:10.1186/s12958-018-0341-2

3. Kristensen DM, Desdoits-Lethimonier C, Mackey AL, et al. Ibuprofen alters human testicular physiology to produce a state of compensated hypogonadism. Proc Natl Acad Sci U S A. 2018;115(4):E715-e724. doi:10.1073/pnas. 1715035115

4. Dohle GR, Smit M, Weber RF. Androgens and male fertility. World J Urol. 2003;21(5):341-345. doi:10.1007/s00345-003-0365-9

5. Corona G, Bianchini S, Sforza A, Vignozzi L, Maggi M. Hypogonadism as a possible link between metabolic diseases and erectile dysfunction in aging men. Hormones (Athens). 2015;14 (4):569-578.

6. O'Hara L, McInnes K, Simitsidellis I, et al. Autocrine androgen action is essential for Leydig cell maturation and function, and protects against late-onset Leydig cell apoptosis in both mice and men. FASEB J. 2015;29(3):894-910. doi:10.1096/fj.14-255729
7. Brinker AM, Raskin I. Determination of triptolide in root extracts of Tripterygium wilfordii by solid-phase extraction and reverse-phase high-performance liquid chromatography. $J$ Chromatogr $A$. 2005;1070(1-2):65-70. doi:10.1016/j.chroma.2005.02.083

8. Xue M, Cheng Y, Han F, et al. Triptolide attenuates renal tubular epithelial-mesenchymal transition via the MiR-188-5p-mediated PI3K/AKT pathway in diabetic kidney disease. Int $J$ Biol Sci. 2018;14(11):1545-1557. doi:10.7150/ijbs.24032

9. Huynh PN, Hikim AP, Wang C, et al. Long-term effects of triptolide on spermatogenesis, epididymal sperm function, and fertility in male rats. J Androl. 2000;21;5:689-699.

10. Lue Y, Sinha Hikim AP, Wang C, et al. Triptolide: a potential male contraceptive. J Androl. 1998;19;4:479-486.

11. Huang R, Li J, Pan F, Zhang B, Yao Y. The activation of GPER inhibits cells proliferation, invasion and EMT of triple-negative breast cancer via CD151/miR-199a-3p bio-axis. Am J Transl Res. 2020;12(1):32-44.

12. Wang Y, Deng X, Dai Y, Niu X, Zhou M. miR-27a downregulation promotes cutaneous squamous cell carcinoma progression via targeting EGFR. Front Oncol. 2019;9:1565. doi:10.3389/fonc.2019.01565

13. Wei J, Yang L, Wu YN, Xu J. Serum miR-1290 and miR-1246 as potential diagnostic biomarkers of human pancreatic cancer. J Cancer. 2020;11(6):1325-1333. doi:10.7150/jca.38048

14. Li L, Zhu Y, Chen T, et al. MiR-125b-2 knockout in testis is associated with targeting to the PAP gene, mitochondrial copy number, and impaired sperm quality. Int J Mol Sci. 2019;20(1).

15. Abu-Halima M, Hammadeh M, Schmitt J, et al. Altered microRNA expression profiles of human spermatozoa in patients with different spermatogenic impairments. Fertil Steril. 2013;99(5):1249-1255. e1216. doi:10.1016/j.fertnstert.2012.11.054

16. Yan X, Ke XX, Zhao H, et al. Triptolide inhibits cell proliferation and tumorigenicity of human neuroblastoma cells. Mol Med Rep. 2015;11 (2):791-796. doi:10.3892/mmr.2014.2814

17. Liang H, Zhang S, Li Z. Ginsenoside Rg3 protects mouse leydig cells against triptolide by downregulation of miR-26a. Drug Des Devel Ther. 2019;13:2057-2066. doi:10.2147/DDDT.S208328

18. Passos JF, Nelson G, Wang C, et al. Feedback between p21 and reactive oxygen production is necessary for cell senescence. Mol Syst Biol. 2010;6:347. doi:10.1038/msb.2010.5

19. Tang L, Li J, Fu W, Wu W, Xu J. Suppression of FADS1 induces ROS generation, cell cycle arrest, and apoptosis in melanocytes: implications for vitiligo. Aging (Albany NY). 2019;11 (24):11829-11843. doi:10.18632/aging.102452

20. Ni B, Jiang Z, Huang X, et al. Male reproductive toxicity and toxicokinetics of triptolide in rats. Arzneimittelforschung. 2008;58 (12):673-680.

21. Hu J, Yu Q, Zhao F, et al. Protection of quercetin against triptolide-induced apoptosis by suppressing oxidative stress in rat Leydig cells. Chem Biol Interact. 2015;240:38-46. doi:10.1016/j. cbi.2015.08.004

22. Sedha S, Kumar S, Shukla S. Role of oxidative stress in male reproductive dysfunctions with reference to phthalate compounds. Urol J. 2015;12(5):2304-2316.

23. Gao S, Wang Z, Zhang C, Jia L, Zhang Y. Oral exposure to atrazine induces oxidative stress and calcium homeostasis disruption in spleen of mice. Oxid Med Cell Longev. 2016;2016:7978219. doi:10.1155/ 2016/7978219

24. Chen Y, Feng X, Hu X, et al. Dexmedetomidine ameliorates acute stress-induced kidney injury by attenuating oxidative stress and apoptosis through inhibition of the ROS/JNK signaling pathway. Oxid Med Cell Longev. 2018;2018:4035310.

25. Wang H, Chen L, Ye XY. [Triptolide induces oxidative stress and apoptosis and activates PIK3/Akt signaling pathway in TM4 sertoli cells]. Beijing Da Хие Хие Baо Yi Хие Ban. 2018;50(4):607-612. Chinese. 
26. Xiong S, Ma W, Jing J, et al. An miR-200 cluster on chromosome 23 regulates sperm motility in zebrafish. Endocrinology. 2018;159 (5):1982-1991. doi:10.1210/en.2018-00015

27. Li P, Chen Y, Juma CA, et al. Differential inhibition of target gene expression by human microRNAs. Cells. 2019;8(8):791. doi:10.3390/ cells8080791

28. Zheng W, Xie W, Yin D, et al. ATG5 and ATG7 induced autophagy interplays with UPR via PERK signaling. Cell Commun Signal. 2019;17(1):42. doi:10.1186/s12964-019-0353-3

29. Codogno P, Meijer AJ. Autophagy and signaling: their role in cell survival and cell death. Cell Death Differ. 2005;12(Suppl 2):1509-1518. doi:10.1038/sj.cdd.4401751

30. Gao F, Li G, Liu C, et al. Autophagy regulates testosterone synthesis by facilitating cholesterol uptake in Leydig cells. J Cell Biol. 2018;217(6):2103-2119. doi:10.1083/jcb.201710078
31. Yahyavy S, Valizadeh A, Saki G, Khorsandi L. Taurine induces autophagy and inhibits oxidative stress in mice Leydig cells. JBRA Assist Reprod. 2020. doi:10.5935/1518-0557.20190079

32. Kim KH, Lee MS. Autophagy-a key player in cellular and body metabolism. Nat Rev Endocrinol. 2014;10(6):322-337. doi:10.1038/ nrendo. 2014.35

33. Kaminskyy VO, Zhivotovsky B. Free radicals in cross talk between autophagy and apoptosis. Antioxid Redox Signal. 2014;21(1):86-102. doi:10.1089/ars.2013.5746

34. Çatakoğlu AB, Kendirci M. Testosterone replacement therapy and cardiovascular events. Turk Kardiyol Dern Ars. 2017;45(7):664-672.

35. Wang Y, Chen F, Ye L, Zirkin B, Chen H. Steroidogenesis in Leydig cells: effects of aging and environmental factors. Reproduction. 2017;154(4):R111-r122. doi:10.1530/REP-17-0064

\section{Publish your work in this journal}

Drug Design, Development and Therapy is an international, peerreviewed open-access journal that spans the spectrum of drug design and development through to clinical applications. Clinical outcomes, patient safety, and programs for the development and effective, safe, and sustained use of medicines are a feature of the journal, which has also been accepted for indexing on PubMed Central. The manuscript management system is completely online and includes a very quick and fair peer-review system, which is all easy to use. Visit http://www. dovepress.com/testimonials.php to read real quotes from published authors. 